\section{계명의대학술지}

\section{Keimyung Medical \\ Journal}

\title{
Ultrasound Guided Infraorbital Nerve Radiofrequency Thermocoagulation in Patients with Trigeminal Neuralgia
}

\author{
Ji Hee Hong, Ho Woo Lee, Sang Baek Kim \\ Department of Anesthesiology and Pain, Keimyung University School of Medicine, Daegu, \\ Korea
}

\begin{abstract}
Trigeminal neuralgia (TN) is a sudden intensive orofacial pain disorder characterized by a unilateral recurrent paroxysmal lancinating pain occurring in the area of trigeminal nerve distribution. Diagnosis is made with characteristic clinical presentations and requires brain magnetic resonance imaging to rule out intracranial tumor or multiple sclerosis which potentially causes secondary TN. Among the various interventional managements, the conventional radiofrequency thermocoagulation (RFT) of Gasserian ganglion results in the highest rate of complete pain relief. We report two cases who presented severe facial pain due to intractable TN of V2 division. For pain relief, Gasserian ganglion RFT was planned. However, both patients could not tolerate the neck extension and chin-up position. Therefore, ultrasound guided infraorbital nerve RFT which does not require such position was performed successfully. When the patients came to the pain clinic 2 weeks later, the numerical rating scale for TN was $2 / 10$ with mild hypoesthesia of V2 division. Infraorbital nerve RFT is an alternative option in patients who is unable to tolerate the ganglion RFT.
\end{abstract}

Keywords: Radio waves, Trigeminal nerve, Trigeminal neuralgia, Ultrasonography

\section{Corresponding Author:}

Ji Hee Hong, MD, PhD

Department of Anesthesiology and Pain

Medicine, Keimyung University School of

Medicine, Dongsan Medical Center, 1035

Dalgubeol-daero, Dalseo-gu, Daegu,

42601, Korea.

Tel: 82-53-258-7767

Fax: 82-53-258-6288

E-mail:swon13@daum.net

(C) 2022 Keimyung University School of Medicine

(c) This is an Open Access article distributed under the terms of the Creative Commons Attribution Non-Commercial License (http://creativecommons. org/licenses/by-nc/4.0/) which permits unrestricted non-commercial use, distribution, and reproduction in any medium, provided the original work is properly cited.

\section{Introduction}

Trigeminal neuralgia (TN) is the most commonly diagnosed form of orofacial pain which usually occurs in 50 to 70 years of age and more frequent in females than in males [1]. TN is characterized by brief episodes of intensive pain, lancinating in nature, occurring recurrently in the area of trigeminal nerve distribution. Pain is triggered by innocuous cutaneous sensations such as chewing, face washing, teeth brushing and talking. Diagnosis is made with characteristic clinical presentations described above and requires brain MRI to rule out intracranial tumor or multiple sclerosis which potentially causes secondary TN [2]. Neurovascular conflict is the leading cause of TN, accounting for 80$90 \%$ of cases, although some patients of TN do not present any neurovascular conflict. An aberrant loop of artery or vein close to the trigeminal nerve root can result in such neurovascular conflict [3,4]. Carbamazepine or oxcarbazepine with gradually increasing doses are usually used to treat TN initially [5]. About $25-30 \%$ of patients either become resistant to the medications or develop unacceptable side effects which eventually requires further interventional management [6]. Among the various interventional managements, the conventional radiofrequency thermocoagulation (RFT) of Gasserian ganglion results in the highest rate of complete pain relief. Over $90 \%$ of patients shows significant pain relief with that treatment [7]. However, various complications have been reported including weakness of masticatory muscles, meningitis, keratitis, anesthesia dolorosa, and intolerable dysesthesia $[8,9]$. Also, it shows sudden in- 
crease of blood pressure or heart rate during the puncture of foramen ovale [10]. Conventional RFT on the Gasserian ganglion requires the position of neck extension to facilitate visualization of foramen ovale. The infraorbital nerve, which is the terminal branch of trigeminal nerve, exits the maxilla via the infraorbital foramen and provides sensory innervation to the lower eyelid, upper lip, and one side of the nose. Recent studies showed that conventional RFT on this peripheral nerve, not the Gasserian ganglion, was effective in relieving the facial pain of TN $[11,12]$. Here, we report two cases of successful infraorbital nerve RFT which were impossible to receive the ganglion RFT due to limited neck extension and severe underlying illness.

\section{Case 1}

A 77-year old man came to the pain clinic complaining of severe recurrent lancinating pain involving right sided upper teeth and gum pain which developed 5 months ago. His pain showed abrupt onset and termination with an electric shock like sensation. The duration of the pain was within 2 minutes. The pain tended to be suddenly triggered by chewing, washing face, and brushing teeth. We assessed that patient as TN based on the characteristic clinical presentation. Brain magnetic resonance imaging (MRI) and magnetic resonance angiography (MRA) findings showed no vascular anomaly, intracranial tumor or any other lesion. He was first treated with $200 \mathrm{mg}$ of carbamazepine twice a day, but it showed minimal efficacy. The numerical rating scale (NRS, 0 being no pain and 10 being the worst pain imaginable) for TN was 9/10. The patient had underlying hypertension, diabetes mellitus, Parkinson's disease, and unilateral leg weakness due to previous cerebral infarction and was taking $50 \mathrm{mg}$ of cilostazol twice a day. He was also previously diagnosed with TN involving right maxillary division 8 years ago.

Ultrasound guided infraorbital nerve blockade was performed twice with one week interval with $0.2 \%$ ropivacaine $0.5 \mathrm{~mL}$ using a hockey stick probe (GE Healthcare, Logiq S8, USA) (Fig. 1). The patient was followed up after 10 days and the NRS score for TN was 6/10 but maintaining normal daily activities was still impossible due to residual pain attacks. The patient was educated to stop cilostazol for 2 days before treatment and was scheduled for the next appointment 10 days later. Since his underlying illness was complicated and severe, we thought that the infraorbital nerve RFT rather than the Gasserian ganglion would be more safe.

The patient lay in the supine position and sterile draping with povidone was done at right midface area. A radiofrequency cannula of 22-gauge, $5 \mathrm{~cm}$, and $5 \mathrm{~mm}$ active tip was used. After confirming the clear visualization of infraorbital foramen, the cannula was inserted toward the infraorbital foramen using an in-plane approach. The cannula was advanced under continuous ultrasound guidance until reaching proximity to the infraorbital nerve and the location of cannula tip was confirmed. Then, the electrical stimulation of $0.3 \mathrm{~V}$ at $50 \mathrm{~Hz}$ frequency were in concordance with the location of the pain. The final position of the cannula tip was modified minutely according to the effect of the stimulation. After successful concordant electrical stimulation in maxillary area, radiofrequency was performed at $70^{\circ} \mathrm{C}$ for 60 seconds for one time. Just after finishing RFT, the patient's vital signs were stable. The patient came to the pain clinic 2 weeks later and the NRS score for TN was $2 / 10$ with mild hypoesthesia involving right maxillary division of trigeminal nerve. After 5 months, the NRS score for TN was still 2/10 with mild hypoesthesia.

\section{Case 2}

An 84-year old man came to the pain clinic complaining of severe recurrent lancinating pain involving right upper gum and cheek which developed one month ago. That patient was medicated with $200 \mathrm{mg}$ of carbamazepine twice a day at our hospital's neurology department and showed alleviation of symptoms for 2 weeks. However, medication showed only minimal efficacy as time goes on. The NRS score for TN was 9/10. We assessed the patient as TN based on the characteristic clinical presentation. Brain MRI and MRA findings showed no vascular anomaly, intracranial tumor or any other lesion. Ultrasound guided infraorbital nerve blockade was performed twice with one week interval using $0.2 \%$ ropivacaine $0.5 \mathrm{~mL}$ (GE Healthcare, Logiq S8, USA) (Fig. 1). The patient was followed up after 7 days and the NRS score for TN was $1 / 10$.

He came to the pain clinic one month later presenting with recurrence of previous symptoms with NRS score 9/10 and was treated again with infraorbital nerve blockade. After this blockade, the NRS decreased to 7, but maintaining normal daily activities was still impossible due to residual pain attacks. RFT of Gasserian ganglion with intravenous anesthesia was planned to relieve his intractable pain. He was monitored with electrocardiography, blood pressure and pulse oximetry.

Facial mask was applied to supply oxygen $(3 \mathrm{~L} / \mathrm{min})$. Patient lay in the supine position with neck extended and chin-up. However, with the position of neck extension and chin-up, 


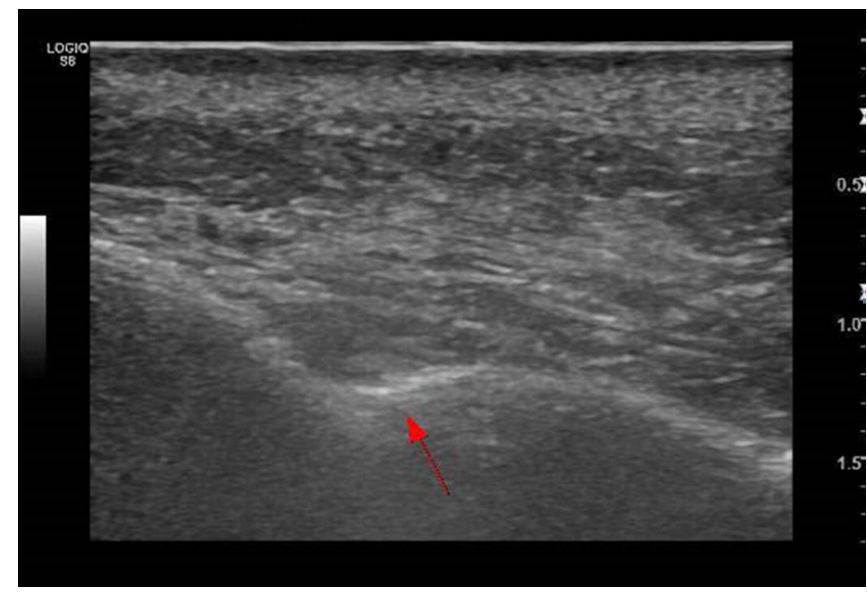

Fig. 1. Ultrasound guided infraorbital nerve blockade. Arrow indicates the infraobital foramen.

the patient felt severe preexisting shoulder pain. This shoulder pain was thought to come from his cervical foraminal stenosis. Since the patient could not bear the position required to the Gasserian ganglion RFT, we could not perform the RFT moreover. Although the ganglion RFT was abandoned due to the occurrence of severe shoulder pain, peripheral nerve RFT was planned to relieve facial pain.

The patient lay in the supine position with sterile draping. A radiofrequency cannula of 22-gauge, $5 \mathrm{~cm}$, and $5 \mathrm{~mm}$ active tip was used. After confirming the clear visualization of infraorbital foramen, the cannula was inserted toward the infraorbital foramen using an in-plane approach. The cannula was advanced under continuous ultrasound guidance until reaching proximity to the infraorbital nerve and the location of cannula tip was confirmed. Then, the electrical stimulation of $0.3 \mathrm{~V}$ at $50 \mathrm{~Hz}$ frequency were in concordance with the location of the pain. The final position of the cannula tip was modified minutely according to the effect of the stimulation. After successful concordant electrical stimulation in maxillary area, radiofrequency was performed at $70^{\circ} \mathrm{C}$ for 60 seconds for one time. Just after finishing RFT, the patient's vital signs were stable. The patient came to the pain clinic 10 days later and the NRS score for TN was $1 / 10$ with mild hypoesthesia around cheek. After 6 months, the NRS score for TN was maintained to $2-3 / 10$.

\section{Discussion}

Two patients of this case report demonstrated significant pain relief after infraorbital nerve RFT. When we tried to perform the ganglion RFT, the first patient had severe underlying illness. The second patient could not bear the neck extension with chin-up position due to the occurrence of shoulder pain. Conventional RFT of Gasserian ganglion under fluoroscopy guidance has been proven to be effective in treating $\mathrm{TN}$ and has been widely used in the treatment of TN in the past decade [9]. The position with neck extension and chin-up is essential for the visualization of foramen ovale. However, such position can be uncomfortable and intolerable in some patients. Neck extension and chin-up can be difficult if the patient has a stiff neck due to ankylosing spondylitis or they can cause unexpected provocation of shoulder pain by aggravating foraminal narrowing just like a patient in this case report. Compared with the ganglion RFT, infraorbital nerve RFT does not require such difficult position. For the confirmation of infraorbital foramen with ultrasound, just lying in bed with neutral position is enough.

Among the procedure steps of ganglion RFT, the puncture of foramen ovale led to significant increase in heart rate $(42 / 48,88 \%)$ and mean arterial pressure $(48 / 48,100 \%)$. Also, the heating stimulation of RFT obviously increased mean arterial pressure and heart rate [10]. Intravenous sedation anesthesia should always be performed during the ganglion RFT since this procedure is very painful and challenging. Considering such unwanted hemodynamic responses and additional intravenous anesthesia during Gasserian ganglion RFT, we thought that first patient who had severe underlying illness would be proper for the infraorbital nerve RFT rather than the ganglion RFT.

The infraorbital RFT has many technical advantages over the ganglion RFT. Moreover, the procedure itself is less challenging and it does not require the intravenous sedation anesthesia. The treatment outcome of the infraorbital nerve RFT showed nearly equivalent efficacy compared with that of the ganglion RFT. Recent study showing the effectiveness of supraorbital nerve RFT has shown 93\% immediate pain relief [12]. Bharti et al. [11] reported that $90 \%$ of patients who received peripheral trigeminal nerve RFT showed successful pain reduction up to 3 months. However, the number of patients who required supplementary medications was more in patients who received peripheral trigeminal nerve RFT than those who received ganglion RFT.

RFT of the peripheral division of trigeminal nerve is considered to produce a conduction block of the irritated nerve axon which results in ultimate pain reduction. RFT of the peripheral division of the trigeminal nerve have been applied for the alleviation of various facial pain [13-15]. RFT of the mental nerve demonstrated significant pain reduction in both pa- 
tients who showed painful neuropathy after tooth extraction [15]. Also, pulsed RFT of the infraorbital nerve in patients of postherpetic neuralgia showed significant pain reduction [13]. RFT of the supraorbital nerve was very effective in relieving the headache of medically intractable hemicranias continua [14].

For the performance of peripheral trigeminal nerve RFT, identification of supraorbital, infraorbital or mental foramen is most important. For such identification, previous studies used fluoroscopy or computed tomogragphy (CT) [11,12]. Foramen ovale is deeply located within the skull base of posterior cranial fossa. Therefore, the identification of foramen ovale has been performed under guidance of fluoroscopy or CT [9]. In contrast to the foramen ovale of skull base, the supraorbital, infraorbital or mental foramen is a superficially located skull foramens [13]. Ultrasound provides good views when we try to identify superficial foramens [13]. In this case report, we used the ultrasound for the confirmation of infraorbital foramen. The possibility of ultrasound guidance during the RFT of infraorbital nerve is another advantage of this procedure with technical easiness compared to the ganglion RFT. However, clinical studies showing good treatment outcome after infraorbital nerve RFT is still lacking compared to Gasserian ganglion RFT.

In conclusion, we could perform the infraorbital nerve RFT successfully in both patients who showed unexpected shoulder pain provocation and with severe underlying illness.

\section{Conflict of interest}

All authors declare no conflicts-of-interest related to this article.

\section{References}

1. De Toledo IP, Conti Reus J, Fernandes M, Porporatti AL, Peres MA, Takaschima A, et al. Prevalence of trigeminal neuralgia: a systematic review. J Am Dent Assoc. 2016;147. 570-6. e2.

2. Brisman R. Trigeminal neuralgia: diagnosis and treatment. World Neurosurg. 2011;76:533-4.

3. Devor M, Amir R, Rappaport ZH. Pathophysiology of trigeminal neuralgia: the ignition hypothesis. Clin J Pain. 2002;18:4-13.

4. Nurmikko T, Eldridge P. Trigeminal neuralgia - pathophysiolo- gy, diagnosis and current treatment. Br J Anaesth. 2001;87:11732.

5. Cruccu G, Gronseth G, Alksne J, Argoff C, Brainin M, Burchiel $\mathrm{K}$, et al. AAN-EFNS guidelines on trigeminal neuralgia management. Eur J Neurol. 2008;15:1013-28.

6. Montano N, Conforti G, Di Bonaventura R, Meglio M, Fernandez E, Papacci F. Advances in diagnosis and treatment of trigeminal neuralgia. Ther Clin Risk Manag. 2015;11:289.

7. Cheng JS, Lim DA, Chang EF, Barbaro NM. A review of percutaneous treatments for trigeminal neuralgia. Oper Neurosurg (Hagerstown). 2014;10:25-33.

8. Kosugi S, Shiotani M, Otsuka Y, Suzuki T, Katori N, Hashiguchi S, et al. Long-term outcomes of percutaneous radiofrequency thermocoagulation of Gasserian ganglion for 2nd-and multiple-division trigeminal neuralgia. Pain Pract. 2015;15:223-8.

9. Kanpolat Y, Savas A, Bekar A, Berk C. Percutaneous controlled radiofrequency trigeminal rhizotomy for the treatment of idiopathic trigeminal neuralgia: 25-year experience with 1,600 patients. Neurosurgery. 2001;48. 524-32. discussion 32-4.

10. Meng Q, Zhang W, Yang Y, Zhou M, Li X. Cardiovascular responses during percutaneous radiofrequency thermocoagulation therapy in primary trigeminal neuralgia. J Neurosurg Anesthesiol. 2008;20:131-5.

11. Bharti N, Sujith J, Singla N, Panda NB, Bala I. Radiofrequency thermoablation of the Gasserian ganglion versus the peripheral branches of the trigeminal nerve for treatment of trigeminal neuralgia: a randomized, control trial. Pain Physician. 2019; 22:147-54

12. Huibin Q, Jianxing L, Guangyu H, Dianen F. The treatment of first division idiopathic trigeminal neuralgia with radiofrequency thermocoagulation of the peripheral branches compared to conventional radiofrequency. J Clin Neurosci. 2009;16:1425-9.

13. Lim SM, Park HL, Moon HY, Kang KH, Kang H, Baek CH, et al. Ultrasound-guided infraorbital nerve pulsed radiofrequency treatment for intractable postherpetic neuralgia - a case report. Korean J Pain. 2013;26:84-8.

14. Weyker P, Webb C, Mathew L. Radiofrequency ablation of the supra-orbital nerve in the treatment algorithm of hemicrania continua. Pain Physician. 2012;15:E719-24.

15. Elahi F, Manolitsis N, Ranganath YS, Reddy C. Mental nerve neuropathy following dental extraction. Pain Physician. 2014; 17:E375-80. 